\title{
Exchange potential via functional differentiation of the Dirac idempotent density matrix
}

\author{
N. H. March ${ }^{1,2}$ and I. A. Howard ${ }^{1}$ \\ ${ }^{1}$ Department of Physics, University of Antwerp, Groenenborgerlaan 171, B-2020 Antwerp, Belgium \\ ${ }^{2}$ Oxford University, Oxford, England
}

(Received 8 December 2003; revised manuscript received 11 February 2004; published 2 June 2004)

\begin{abstract}
Following two illustrative examples, a quite general form for the functional derivative of the Dirac density matrix $\gamma\left(\mathbf{r}_{1}, \mathbf{r}_{2}\right)$ with respect to the electron density $\rho(\mathbf{r})$ is proposed. In turn, this functional derivative is related to a further three-point object, which reduces to the linear response function $\chi_{0}\left(\mathbf{r}_{1}, \mathbf{r}\right)$ when $\mathbf{r}_{2}$ tends to $\mathbf{r}_{1}$. The main application is then to derive the exchange-only potential of density functional theory. To avoid heavy numerical work in molecules and clusters, an approximation is suggested, with major calculational simplifications as a probable consequence.
\end{abstract}

DOI: 10.1103/PhysRevA.69.064101

PACS number(s): 03.65.- w, 71.15.Mb

Considerable current interest is being shown in the firstprinciples treatment of the exchange-only potential $V_{x}(\mathbf{r})$ of density functional theory (DFT). Thus, the work of Della Sala and Görling [1-3], that of the present writers [4], and numerical calculations on atomic ions [5] using the optimized effective potential (OEP) of Talman and Shadwick [6] may be cited in the above context. In this Brief Report, we shall take as the starting point the Dirac exchange energy [7] $-E_{x}$, say—in terms of the idempotent Dirac density matrix $\gamma\left(\mathbf{r}, \mathbf{r}^{\prime}\right)$. Dirac's result is

$$
E_{x}=-\frac{e^{2}}{4} \int \frac{\gamma\left(\mathbf{r}_{1}, \mathbf{r}_{2}\right)^{2}}{\left|\mathbf{r}_{1}-\mathbf{r}_{2}\right|} d \mathbf{r}_{1} d \mathbf{r}_{2} .
$$

To gain insight into what follows, let us first consider a twoelectron spin-compensated system-say, $\mathrm{He}$ or $\mathrm{H}_{2}$-in its ground state with electron density $\rho(\mathbf{r})$.

Then the Dirac density matrix takes the elementary form

$$
\gamma\left(\mathbf{r}_{1}, \mathbf{r}_{2}\right)=\rho\left(\mathbf{r}_{1}\right)^{1 / 2} \rho\left(\mathbf{r}_{2}\right)^{1 / 2},
$$

and we can immediately functionally differentiate to obtain a prime focus of this article: namely, the functional derivative $\delta \gamma\left(\mathbf{r}_{1}, \mathbf{r}_{2}\right) / \delta \rho(\mathbf{r})$. The result following by an elementary calculation from Eq. (2) is

$$
\frac{\delta \gamma\left(\mathbf{r}_{1}, \mathbf{r}_{2}\right)}{\delta \rho(\mathbf{r})}=\frac{1}{2}\left\{\frac{\rho\left(\mathbf{r}_{2}\right)}{\rho\left(\mathbf{r}_{1}\right)}\right\}^{1 / 2} \delta\left(\mathbf{r}_{1}-\mathbf{r}\right)+\frac{1}{2}\left\{\frac{\rho\left(\mathbf{r}_{1}\right)}{\rho\left(\mathbf{r}_{2}\right)}\right\}^{1 / 2} \delta\left(\mathbf{r}_{2}-\mathbf{r}\right) .
$$

If we construct from Eq. (1) the exchange potential $V_{x}(\mathbf{r})$ as the functional derivative of $E_{x}[8]$ —namely,

$$
V_{x}(\mathbf{r})=\frac{\delta E_{x}}{\delta \rho(\mathbf{r})},
$$

then we find

$$
V_{x}(\mathbf{r})=-\frac{e^{2}}{2} \int \frac{\gamma\left(\mathbf{r}_{1}, \mathbf{r}_{2}\right)}{\left|\mathbf{r}_{1}-\mathbf{r}_{2}\right|} \frac{\delta \gamma\left(\mathbf{r}_{1}, \mathbf{r}_{2}\right)}{\delta \rho(\mathbf{r})} d \mathbf{r}_{1} d \mathbf{r}_{2} .
$$

As a first, and elementary, application of this result (5), we insert the two-electron result (3) to find

$$
\begin{aligned}
V_{x}(\mathbf{r})= & -\frac{e^{2}}{4} \int \frac{\gamma\left(\mathbf{r}, \mathbf{r}_{2}\right)}{\left|\mathbf{r}-\mathbf{r}_{2}\right|}\left\{\frac{\rho\left(\mathbf{r}_{2}\right)}{\rho(\mathbf{r})}\right\}^{1 / 2} d \mathbf{r}_{2} \\
& -\frac{e^{2}}{4} \int \frac{\gamma\left(\mathbf{r}_{1}, \mathbf{r}\right)}{\left|\mathbf{r}-\mathbf{r}_{1}\right|}\left\{\frac{\rho\left(\mathbf{r}_{1}\right)}{\rho(\mathbf{r})}\right\}^{1 / 2} d \mathbf{r}_{1} .
\end{aligned}
$$

Using again $\gamma$ from Eq. (2) we find, almost immediately,

$$
V_{x}(\mathbf{r})=-\frac{e^{2}}{4} \int \frac{\rho\left(\mathbf{r}_{2}\right)}{\left|\mathbf{r}-\mathbf{r}_{2}\right|} d \mathbf{r}_{2}-\frac{e^{2}}{4} \int \frac{\rho\left(\mathbf{r}_{1}\right)}{\left|\mathbf{r}-\mathbf{r}_{1}\right|} d \mathbf{r}_{1}=-\frac{1}{2} V_{e l}(\mathbf{r}) .
$$

A special case of this result is already given for the twoelectron Hookean atom by Kais et al. [9], who, however, bypassed the functional derivative route emphasized here.

Turning to our second example - the Be atom or the $\mathrm{H}_{2}$ dimer-we follow Dawson and March [10] in writing $\gamma\left(\mathbf{r}_{1}, \mathbf{r}_{2}\right)$ in terms of the density amplitude $\rho^{1 / 2}$ again, but now including the phase $\theta(\mathbf{r})$, which yields, for the density matrix,

$$
\gamma\left(\mathbf{r}_{1}, \mathbf{r}_{2}\right)=\rho\left(\mathbf{r}_{1}\right)^{1 / 2} \rho\left(\mathbf{r}_{2}\right)^{1 / 2} \cos \left[\theta\left(\mathbf{r}_{1}\right)-\theta\left(\mathbf{r}_{2}\right)\right] .
$$

Employing the equation of motion for the density matrix, it readily follows that the force $-\partial V / \partial r$ corresponding to the one-body potential $V(r)$ is related to $\gamma\left(\mathbf{r}, \mathbf{r}^{\prime}\right)$ by

$$
\begin{aligned}
\frac{\partial V}{\partial r}= & \frac{\partial}{\partial r}\left[\frac{\nabla_{r}^{2} \gamma-\nabla_{r^{\prime}}^{2} \gamma}{2 \gamma}\right]=-2 \theta^{\prime} \theta^{\prime \prime}-\left[\frac{2}{r}+\frac{\rho^{\prime}}{\rho}\right] \theta^{\prime 2}+\frac{\rho^{\prime \prime \prime}}{4 \rho} \\
& +\frac{\rho^{\prime \prime}}{2 r \rho}-\frac{\rho^{\prime} \rho^{\prime \prime}}{2 \rho^{2}}-\frac{\rho^{\prime}}{2 r^{2} \rho}-\frac{\rho^{\prime 2}}{2 r \rho^{2}}+\frac{\rho^{\prime 3}}{4 \rho^{3}}
\end{aligned}
$$

or

$$
\frac{\partial V}{\partial r}=-\frac{\partial}{\partial r}\left[\frac{\delta T_{W}}{\delta \rho(r)}\right]+\frac{\partial V_{\Delta}}{\partial r},
$$

where $T_{W}$ is the von Weizsäcker [11] inhomogeneity kinetic energy $\int(\nabla \rho)^{2} /(8 \rho) d \mathbf{r}$ and $V_{\Delta}$ is the "correction" to the potential contribution $-\delta T_{W} / \delta \rho(r)$. Then it follows from Eqs. (9) and (10) that 


$$
\frac{\partial V_{\Delta}}{\partial r}=-2 \theta^{\prime} \theta^{\prime}-\left[\frac{2}{r}+\frac{\rho^{\prime}}{\rho}\right] \theta^{\prime 2} .
$$

But following Dawson and March [10] one has the pendulum equation relating density $\rho$ and phase $\theta$ :

$$
\theta^{\prime \prime}+\frac{\left(r^{2} \rho\right)^{\prime}}{r^{2} \rho} \theta^{\prime}=2 \xi \sin (2 \theta),
$$

where $\xi=\left(\epsilon_{1}-\epsilon_{2}\right) / 2$, with $\epsilon_{1}$ and $\epsilon_{2}$ the $1 s$ and $2 s$ eigenvalues generated by $V(r)$. Integration of Eq. (11) and use of the pendulum equation (12) plus its first derivative, then yields

$$
V_{\Delta}(r)=-\frac{\theta^{\prime 2}}{2}+\frac{\theta^{\prime \prime}}{4 \theta^{\prime}}+\frac{\theta^{\prime \prime}}{4 \theta^{\prime}}\left(\frac{2}{r}+\frac{\rho^{\prime}}{\rho}\right)-\frac{1}{4}\left[\frac{2}{r^{2}}-\frac{\rho^{\prime \prime}}{\rho}+\frac{\left(\rho^{\prime}\right)^{2}}{\rho^{2}}\right] .
$$

We note that the above allows an exact functional differentiation of the single-particle kinetic energy, since $V$ $=-\delta T_{s} / \delta \rho(r)$ to within an additive constant.

In connection with the study of Kleinman [12] on Slater's nonlocal potential [13]—namely,

$$
V_{x}^{S l}(\mathbf{r})=-\frac{e^{2}}{2 \rho(\mathbf{r})} \int \frac{\gamma\left(\mathbf{r}, \mathbf{r}_{2}\right)^{2}}{\left|\mathbf{r}-\mathbf{r}_{2}\right|} d \mathbf{r}_{2},
$$

Holas and March [14] derived the exchange potential in terms of $\rho(\mathbf{r})$ and $\theta(\mathbf{r})$, their result correcting the Slater potential by a term

$$
V_{x}(\mathbf{r})-V_{x}^{S l}(\mathbf{r})=F[\theta(\mathbf{r})],
$$

without functional derivatives entering, which parallels the above example of functional differentiation of $T_{s}[\rho]$ for $E_{x}[\rho]$. For the two-level atomic ion with atomic number $Z$, we may write

$$
V_{x}=V+\frac{Z}{r}-V_{e s}
$$

where the electrostatic potential $V_{e s}$ is given by

$$
V_{e s}=-\int^{r} \frac{Q\left(r^{\prime}\right)}{r^{\prime 2}} d r^{\prime}: Q(r)=4 \pi \int_{0}^{r} r^{2} \rho(r) d r,
$$

so that the exchange potential may be determined as

$$
\left(r V_{x}\right)^{\prime}=\frac{\partial}{\partial r}\left[r\left(V_{\Delta}-\frac{\delta T_{W}}{\delta \rho}\right)\right]+\frac{\partial}{\partial r}\left[r \int^{r} \frac{Q\left(r^{\prime}\right)}{r^{\prime 2}} d r^{\prime}\right] .
$$

Note, however, that we have here bypassed the functional derivatives (4) and (5) by appealing directly to Eqs. (8) and (9), a procedure so far carried out only for a two-level atomic ion. We shall return, albeit briefly, to the two-level example based on the density matrix (8) below.

To obtain a general formula for the functional derivatives in Eqs. (4) and (5), following the above specific examples, we next appeal to the exchange potential derived by Shaginyan [15]. His starting point is an alternative to the Dirac form of $E_{x}$, given for example by Pines and Nozieres [16]. Their result involves the frequency-dependent linear response function $\chi_{0}\left(\mathbf{r}_{1}, \mathbf{r}_{2}, \omega\right)$ and reads

$$
\begin{aligned}
E_{x}= & -\frac{1}{2} \int\left[\operatorname{Im} \chi_{0}\left(\mathbf{r}_{1}, \mathbf{r}_{2}, \omega\right)+\pi \rho\left(\mathbf{r}_{1}\right) \delta\left(\mathbf{r}_{1}-\mathbf{r}_{2}\right) \delta(\omega)\right] \\
& \times \frac{e^{2}}{\left|\mathbf{r}_{1}-\mathbf{r}_{2}\right|} d \mathbf{r}_{1} d \mathbf{r}_{2} \frac{d \omega}{\pi}
\end{aligned}
$$

Whereas it is natural from Eq. (1), but not of course unique, to define an exchange energy density $\epsilon_{x}(\mathbf{r})$ as the negative definite quantity

$$
\boldsymbol{\epsilon}_{x}(\mathbf{r})=-\frac{e^{2}}{4} \int \frac{\gamma\left(\mathbf{r}, \mathbf{r}^{\prime}\right)^{2}}{\left|\mathbf{r}-\mathbf{r}^{\prime}\right|} d \mathbf{r}^{\prime}
$$

a possible definition from Eq. (19) would be

$$
\begin{aligned}
\epsilon_{x}^{S h}(\mathbf{r})= & -\frac{1}{2} \int\left[\operatorname{Im} \chi_{0}\left(\mathbf{r}, \mathbf{r}_{2}, \omega\right)+\pi \rho(\mathbf{r}) \delta\left(\mathbf{r}-\mathbf{r}_{2}\right) \delta(\omega)\right] \\
& \times \frac{e^{2}}{\left|\mathbf{r}-\mathbf{r}_{2}\right|} d \mathbf{r}_{2} \frac{d \omega}{\pi}
\end{aligned}
$$

It is then clear that $\epsilon_{x}^{S h}(\mathbf{r})$ following from the expression of Shaginyan [15] taken as starting point can differ from the definition of $\epsilon_{x}(\mathbf{r})$ in Eq. (20) only by a function-say, $\Delta(\mathbf{r})=\epsilon_{x}(\mathbf{r})-\epsilon_{x}^{S h}(\mathbf{r})$ - which satisfies

$$
\int \Delta(\mathbf{r}) d \mathbf{r}=0
$$

since Eqs. (20) and (21) are both constructed to yield, by volume integration, the same total exchange energy $E_{x}$. The Slater potential given in Eq. (14) is equivalently written using Eq. (20) as

$$
V_{x}^{S l}(\mathbf{r})=\frac{2 \epsilon_{x}(\mathbf{r})}{\rho(\mathbf{r})},
$$

or as

$$
V_{x}^{S l}(\mathbf{r})=\frac{2 \epsilon_{x}^{S h}(\mathbf{r})}{\rho(\mathbf{r})}+\frac{2 \Delta(\mathbf{r})}{\rho(\mathbf{r})}
$$

While $V_{x}^{S l}(\mathbf{r})$ given by Eq. (23) is clearly finite everywhere, it may be that singularities appear in the two pieces separately in Eq. (24), though then these must of course cancel in the sum. The merit of Eq. (21) is, as shown by Shaginyan [15], that the functional differentiation involved in Eq. (4) can be carried out using Eq. (21).

Let us then return to the functional derivative $\delta \gamma\left(\mathbf{r}_{1}, \mathbf{r}_{2}\right) / \delta_{\rho}(\mathbf{r})$ focused on earlier in Eqs. (3) and (5). We can extract a proposed form for this from Eq. (9) of the study of Shaginyan [15]. This may be expressed as 


$$
\begin{aligned}
\frac{\delta \gamma\left(\mathbf{r}_{1}, \mathbf{r}_{2}\right)}{\delta \rho(\mathbf{r})}= & \int d \mathbf{r}^{\prime} \chi_{0}^{-1}\left(\mathbf{r}, \mathbf{r}^{\prime}\right) \sum_{i}\left[\phi_{i}\left(\mathbf{r}^{\prime}\right) \varphi_{i}^{*}\left(\mathbf{r}_{1}\right) G\left(\mathbf{r}_{2}, \mathbf{r}^{\prime}, \epsilon_{i}\right)\right. \\
& \left.+\phi_{i}^{*}\left(\mathbf{r}^{\prime}\right) \phi_{i}\left(\mathbf{r}_{1}\right) G^{*}\left(\mathbf{r}_{2}, \mathbf{r}^{\prime}, \epsilon_{i}\right)\right] \\
= & \int d \mathbf{r}^{\prime} \chi_{0}^{-1}\left(\mathbf{r}, \mathbf{r}^{\prime}\right) F\left(\mathbf{r}^{\prime}, \mathbf{r}_{1}, \mathbf{r}_{2}\right),
\end{aligned}
$$

where $\phi_{i}(\mathbf{r})$ and $\epsilon_{i}$ denote Kohn-Sham orbitals and eigenvalues while $G$ is the single-particle Green function defined by $G\left(\mathbf{r}, \mathbf{r}^{\prime}\right)=\sum \phi_{i}(\mathbf{r}) \phi_{i}^{*}\left(\mathbf{r}^{\prime}\right) /\left(\epsilon-\epsilon_{i}+i \delta\right)$ [15]. Together with Eq. (5), this equation (25) is one of the central results of this Brief Report. A further important result follows almost immediately from the definition of the function $F\left(\mathbf{r}^{\prime}, \mathbf{r}_{1}, \mathbf{r}_{2}\right)$ in Eq. (25). Taking the limit $\mathbf{r}_{2} \rightarrow \mathbf{r}_{1}$, it is easy to show that $F$ has the attractive property that

$$
F\left(\mathbf{r}^{\prime}, \mathbf{r}_{1}, \mathbf{r}_{2}\right)_{\mathbf{r}_{2}=\mathbf{r}_{1}}=\chi_{0}\left(\mathbf{r}^{\prime}, \mathbf{r}_{1}\right),
$$

where we recall that $\chi_{0}$ is the linear response function. This is especially significant because (a) $\chi_{0}^{-1}\left(\mathbf{r}, \mathbf{r}^{\prime}\right)$ also enters Eq. (25) and (b) one has the property [15]

$$
\int d \mathbf{r}^{\prime} \chi_{0}^{-1}\left(\mathbf{r}, \mathbf{r}^{\prime}\right) d \mathbf{r}^{\prime} \chi_{0}\left(\mathbf{r}^{\prime}, \mathbf{r}_{1}\right)=\delta\left(\mathbf{r}-\mathbf{r}_{1}\right) .
$$

These results (25)-(27) lead us to what seems a natural enough step: namely, to the approximate factorization embodied by writing

$$
F\left(\mathbf{r}^{\prime}, \mathbf{r}_{1}, \mathbf{r}_{2}\right)=\chi_{0}\left(\mathbf{r}^{\prime}, \mathbf{r}_{1}\right) f\left(\mathbf{r}_{1}, \mathbf{r}_{2}\right),
$$

where

$$
f\left(\mathbf{r}_{1}, \mathbf{r}_{1}\right)=1 .
$$

Inserting Eq. (28) into Eq. (25) allows integration over $\mathbf{r}^{\prime}$ to be accomplished using Eq. (27) to yield

$$
\frac{\delta \gamma\left(\mathbf{r}_{1}, \mathbf{r}_{2}\right)}{\delta \rho(\mathbf{r})}=\delta\left(\mathbf{r}-\mathbf{r}_{1}\right) f\left(\mathbf{r}_{1}, \mathbf{r}_{2}\right) .
$$

However, there exists one defect of the factorization (28). If one takes the functional derivative of the Dirac density matrix with respect to the density on both sides of the idempotency identity, everything is of course valid still if one uses the exact result (25). In contrast, if one employs Eq. (30), the precise identity is sacrificed. Notwithstanding this, though $f\left(\mathbf{r}_{1}, \mathbf{r}_{2}\right)$ should eventually be chosen to minimize departures from idempotency requirements, we deem it still worthwhile, motivated by the first example, particularly in Eq. (3), to "symmetrize" Eq. (30) to propose the following form for the approximate functional derivative:

$$
\frac{\delta \gamma\left(\mathbf{r}_{1}, \mathbf{r}_{2}\right)}{\delta \rho(\mathbf{r})}=\frac{1}{2} \delta\left(\mathbf{r}-\mathbf{r}_{1}\right) f\left(\mathbf{r}_{1}, \mathbf{r}_{2}\right)+\frac{1}{2} \delta\left(\mathbf{r}-\mathbf{r}_{2}\right) f\left(\mathbf{r}_{2}, \mathbf{r}_{1}\right) .
$$

It is tempting, from the two-electron example in Eq. (3), to assume that $f\left(\mathbf{r}_{1}, \mathbf{r}_{2}\right)$ may be modelled in terms of $\gamma\left(\mathbf{r}_{1}, \mathbf{r}_{2}\right)$ and $\rho\left(\mathbf{r}_{1}\right)$. Thus Eq. (3) is readily rewritten by employing Eq. (2) as

$$
\frac{\delta \gamma\left(\mathbf{r}_{1}, \mathbf{r}_{2}\right)}{\delta \rho(\mathbf{r})}=\frac{1}{2} \frac{\gamma\left(\mathbf{r}_{1}, \mathbf{r}_{2}\right)}{\rho\left(\mathbf{r}_{1}\right)} \delta\left(\mathbf{r}_{1}-\mathbf{r}\right)+\frac{1}{2} \frac{\gamma\left(\mathbf{r}_{2}, \mathbf{r}_{1}\right)}{\rho\left(\mathbf{r}_{2}\right)} \delta\left(\mathbf{r}_{2}-\mathbf{r}\right),
$$

and evidently in this example therefore Eq. (31) corresponds to the exact form (32) if $f\left(\mathbf{r}_{1}, \mathbf{r}_{2}\right)=\gamma\left(\mathbf{r}_{1}, \mathbf{r}_{2}\right) / \rho\left(\mathbf{r}_{1}\right)$.

In fact, this form of $f\left(\mathbf{r}_{1}, \mathbf{r}_{2}\right)$ leads back quite generally to the Slater potential $V_{x}^{S l}(\mathbf{r})$ in Eq. (14), which Kleinman has stressed is a "partial" functional derivative of $E_{x}$ in Eq. (1) with respect to $\rho(\mathbf{r})$. As Holas and March [14] point out, in the two-level case one must include in the functional derivative of Eq. (1) the phase angle $\theta(\mathbf{r})$, which then yields calculable corrections to the Slater potential, which is, however, already a very useful starting approximation for $V_{x}(r)$ for the two-level case of the Be atom. A "correction" to the approximation $f\left(\mathbf{r}_{1}, \mathbf{r}_{2}\right) \sim \gamma\left(\mathbf{r}_{1}, \mathbf{r}_{2}\right) / \rho\left(\mathbf{r}_{1}\right)$ which preserves Eq. (29) and has the form $\lambda(N)\left|\mathbf{r}_{1}-\mathbf{r}_{2}\right| V_{x}\left(\mathbf{r}_{1}\right) \gamma\left(\mathbf{r}_{1}, \mathbf{r}_{2}\right) / \rho\left(\mathbf{r}_{1}\right)$ suggests itself; this can be shown to establish then approximate contact with Refs. [1-4]. However, $\lambda(N)$, with $N$ the number of occupied levels, would need to be found by some "least squares" minimization of the departure from idempotency requirements, but it would take us too far from our main theme to go into further details along these lines.

In summary, the main results of this Brief Report are embodied in Eq. (25) for the functional derivative of the Dirac density matrix $\gamma\left(\mathbf{r}_{1}, \mathbf{r}_{2}\right)$ with respect to the electron density $\rho(\mathbf{r})$ and Eq. (5) for the exchange potential. The function $F\left(\mathbf{r}^{\prime}, \mathbf{r}_{1}, \mathbf{r}_{2}\right)$ thereby introduced reduces on the diagonal $\mathbf{r}_{2}$ $=\mathbf{r}_{1}$ to the linear response function $\chi_{0}\left(\mathbf{r}^{\prime}, \mathbf{r}_{1}\right)$, which is appealing since its inverse in the form $\chi_{0}^{-1}\left(\mathbf{r}, \mathbf{r}^{\prime}\right)$ also enters Eq. (25). Further work on the off-diagonal properties of the three-point function $F$ may be instructive for the future, by both analytical and numerical routes. It could be especially important if useful approximations to $F$ could be found which involved only occupied Kohn-Sham orbitals in contrast to the present form defined in Eq. (25).

I.A.H. acknowledges support by the IWT-Flemish region under Grant No. IWT-161.
[1] F. Della Sala and A. Görling, J. Chem. Phys. 115, 5718 (2001).

[2] O. V. Gritsenko and E. J. Baerends, Phys. Rev. A 64, 042506 (2001).

[3] See also M. Grüning, O. V. Gritsenko, and E. J. Baerends, J.
Chem. Phys. 116, 6435 (2002).

[4] I. A. Howard and N. H. March, J. Chem. Phys. 119, 5789 (2003).

[5] I. A. Howard, N. H. March, and J. D. Talman, Phys. Rev. A 68, 044502 (2003). 
[6] J. D. Talman and W. F. Shadwick, Phys. Rev. A 14, 36 (1976). [7] P. A.M. Dirac, Proc. Cambridge Philos. Soc. 26, 376 (1930). [8] See, for example, R. G. Parr and W. Yang, Density Functional Theory of Atoms and Molecules (Oxford University Press, Oxford, 1989).

[9] S. Kais, D. R. Herschbach, and R. D. Levine, J. Chem. Phys. 91, 7791 (1989).

[10] K. A. Dawson and N. H. March, J. Chem. Phys. 81, 5850
(1984).

[11] C. F. Weizsäcker, Z. Phys. 96, 341 (1935).

[12] L. Kleinman, Phys. Rev. B 49, 14197 (1994).

[13] J. C. Slater, Phys. Rev. 81, 385 (1951).

[14] A. Holas and N. H. March, Phys. Rev. B 55, 1295 (1997).

[15] V. R. Shaginyan, Phys. Rev. A 47, 1507 (1993).

[16] D. Pines and P. Nozieres, The Theory of Quantum Liquids (Benjamin, New York, 1966), Vol. 1. 